\title{
THE ANATOMY OF STAR FORMATION IN NGC 300
}

\author{
G. Helou, ${ }^{1}$ H. Roussel ${ }^{2}$ P. Appleton, ${ }^{1}$ D. Frayer, ${ }^{1}$ S. Stolovy, ${ }^{1}$ L. Storrie-Lombardi, ${ }^{1}$ R. Hurt, ${ }^{1}$ P. Lowrance, ${ }^{1}$ \\ D. Makovoz, ${ }^{1}$ F. Masci, ${ }^{1}$ J. Surace, ${ }^{1}$ K. D. Gordon, ${ }^{3}$ A. Alonso-Herrero, ${ }^{3}$ C. W. Engelbracht, ${ }^{3}$ K. Misselt, ${ }^{3}$ \\ G. Rieke, ${ }^{3}$ M. Rieke, ${ }^{3}$ S. P. Willner, ${ }^{4}$ M. Pahre, ${ }^{4}$ M. L. N. Ashby, ${ }^{4}$ G. G. Fazio, ${ }^{4}$ and H. A. Smith ${ }^{4}$ \\ Received 2004 March 30; accepted 2004 May 5
}

\begin{abstract}
The Spitzer Space Telescope was used to study the mid- to far-infrared properties of NGC 300 and to compare dust emission to $\mathrm{H} \alpha$ to elucidate the heating of the interstellar medium (ISM) and the star formation cycle at scales smaller than $100 \mathrm{pc}$. The new data allow us to discern clear differences in the spatial distribution of $8 \mu \mathrm{m}$ dust emission with respect to $24 \mu \mathrm{m}$ dust and to $\mathrm{H}$ II regions traced by $\mathrm{H} \alpha$ light. The $8 \mu \mathrm{m}$ emission highlights the rims of $\mathrm{H}$ II regions, and the $24 \mu \mathrm{m}$ emission is more strongly peaked in star-forming regions than $8 \mu \mathrm{m}$. We confirm the existence and approximate amplitude of interstellar dust emission at $4.5 \mu \mathrm{m}$, detected statistically in Infrared Space Observatory (ISO) data, and conclude it arises in star-forming regions. When averaging over regions larger than $\sim 1 \mathrm{kpc}$, the ratio of $\mathrm{H} \alpha$ to aromatic feature emission in NGC 300 is consistent with the values observed in disks of spiral galaxies. The mid- to far-infrared spectral energy distribution of dust emission is generally consistent with pre-Spitzer models.
\end{abstract}

Subject headings: galaxies: individual (NGC 300) — galaxies: ISM — infrared: galaxies — stars: formation

Online material: color figure

\section{INTRODUCTION}

NGC 300 is a SA(s)d galaxy in the Sculptor group of galaxies, at a distance of about 2.1 Mpc (Freedman et al. 1992), viewed at an inclination of about $50^{\circ}$ (Puche et al. 1990). Its total luminosities in the blue band (3855-4985 $\AA$ ) and in the far-infrared (42.5-122.5 $\mu \mathrm{m}$, estimated from Spitzer Space Telescope data) are $\sim 3.3 \times 10^{8}$ and $2.2 \times 10^{8} L_{\odot}$, bol, respectively. Its $L_{\mathrm{FIR}} / L_{B}$ ratio is thus very close to the average ratio for the blue magnitude-limited sample studied by Thuan \& Sauvage (1992). Because of its large angular extent and low surface brightness, no reliable total radio continuum flux measurement exists (only discrete sources are detected). NGC 300 has a striking appearance in the visible and in $\mathrm{H} \alpha$ due to several $\mathrm{H}$ II regions with nearly circular shapes and various degrees of filling-in (Deharveng et al. 1988; Hoopes et al. 1996; see Fig. 1 [Plate 1]). Its large H I envelope (Puche et al. 1990) extends well beyond the visible image. Pannuti et al. (2000) reported a total of 44 supernova remnant (SNR) candidates, evidence that the current star formation activity manifested as $\mathrm{H}$ II regions has been ongoing for tens of millions of years (see also Butler et al. 2004). The gas-phase oxygen abundance varies between about 1.4 and 0.4 times the solar value across the disk of the galaxy (Deharveng et al. 1988). Because of its proximity, NGC 300 allows Spitzer (Werner et al. 2004) to compare dust emission to other interstellar medium (ISM) components and discern the interplay between the ISM and the star formation cycle at scales smaller than $100 \mathrm{pc}$.

\section{OBSERVATIONS AND DATA REDUCTION}

The Infrared Array Camera (IRAC; Fazio et al. 2004) observations of NGC 300 are $12 \mathrm{~s}$ frames and map the galaxy

\footnotetext{
${ }^{1}$ Spitzer Science Center, California Institute of Technology, MC 220-6, Pasadena, CA 91125.

${ }^{2}$ California Institute of Technology, MC 320-47, Pasadena, CA 91125.

3 Steward Observatory, University of Arizona, Tucson, AZ 85721.

4 Harvard-Smithsonian Center for Astrophysics, 60 Garden Street, Cambridge, MA 02138.
}

in approximately half-array spacings, yielding a total time per sky position of $48 \mathrm{~s}$. They were reduced with the standard Spitzer Science Center data reduction pipeline (ver. 9.5). Because of the readout of bright point sources, the signal was reduced (pulled down) in some columns of the array; ${ }^{5}$ a correction measured from the map was applied. Persistent images left by bright sources were found by median-combining all the dithered positions together and identifying any remaining sources. These objects were removed from individual frames. The data were then combined into a mosaic using a cosmic-ray rejection and a background matching applied between overlapping fields of view. The relative photometric uncertainty is of the order of $5 \%$, and the absolute uncertainty is $10 \%$; uncertainty tied to the angular sizes of source and measuring aperture contributes up to $15 \%$.

Images of NGC 300 at 24, 70, and $160 \mu \mathrm{m}$ were obtained with the Multiband Imaging Photometer for Spitzer (MIPS; Rieke et al. 2004) in the scan-map mode. The final mosaics have a total exposure time of approximately 160,80 , and $16 \mathrm{~s}$ per point at 24,70 , and $160 \mu \mathrm{m}$, respectively. The MIPS images were reduced using the MIPS Instrument Team Data Analysis Tool (Gordon et al. 2004) as described by Engelbracht et al. (2004). The uncertainties on the final absolute calibrations are estimated at $10 \%, 20 \%$, and $20 \%$ for the 24,70 , and $160 \mu \mathrm{m}$ data, respectively. The 70 and $160 \mu \mathrm{m}$ images exhibit linear streaks along the scan direction, which are residual instrumental artifacts due to the time-dependent responsivity of the Ge detectors and affect the photometry in large apertures.

The H $\alpha(6563 \AA)+\left[\mathrm{N}_{\text {II }}\right](6583$ and $6548 \AA)$ map was derived from images posted in the NASA/IPAC Extragalactic Database by Larsen \& Richtler (1999). The narrowband image containing $\mathrm{H} \alpha$ emission and the $R$-band image were aligned and rescaled to subtract the stellar continuum, deriving the scaling factor from aperture photometry on 32 bright stars. Residuals from saturated or improperly subtracted stars were

\footnotetext{
${ }^{5}$ See the Spitzer Observer's Manual at http://ssc.spitzer.caltech.edu/documents/som/.
} 


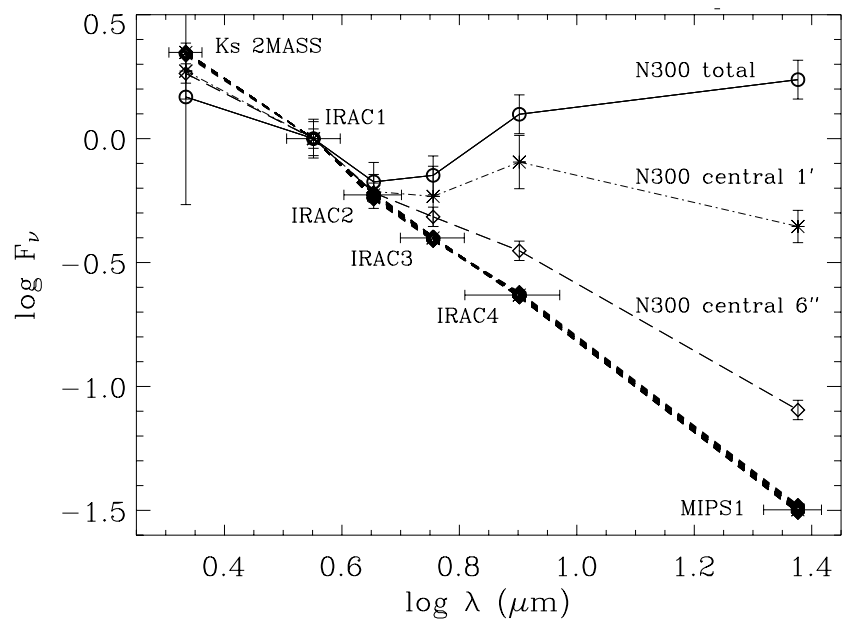

FIG. 2.-SED decomposition. The SEDs of NGC 300 in three different apertures are shown, with all fluxes normalized to $3.6 \mu \mathrm{m}$ : the nuclear stellar cluster (clearly contaminated by dust emission judging from morphology in the IRAC maps), the inner arcminute, where the dust contribution is relatively small, and the whole galaxy. The thick dashed lines trace model photospheric SEDs from 2 to $24 \mu \mathrm{m}$, derived from Starburst99 for a range of star formation histories with a Salpeter IMF and two metallicities, $Z=0.008$ and $0.02 Z_{\odot}$, and transformed to IRAC filter fluxes. The horizontal error bars represent the filter widths at half-maximum transmission. The $K_{s}$ fluxes are lower limits, because the Two Micron All Sky Survey map is not sensitive to the diffuse emission.

masked out, and the final image was regridded and rotated to match the IRAC reference frame.

The Spitzer and $\mathrm{H} \alpha+\left[\mathrm{N}_{\mathrm{II}}\right]$ images are shown in Figure 1. The images contain three very bright Milky Way stars directly superposed on the disk. We masked them out in the IRAC and $24 \mu \mathrm{m}$ images, as well as additional point sources, as described in $\S 3.1$ below (Fig. $4 b$ ). Foreground stars will be removed more rigorously in a later paper. Fainter, indistinguishable stars are unlikely to affect significantly the results presented here.

\section{PHYSICAL CONTENT OF MID-INFRARED IMAGES}

Whereas at $\lambda \geq 24 \mu \mathrm{m}$ the emission is dominated by interstellar dust, the mid-infrared marks the transition from stellar to interstellar emission. The appearance of the galaxy shifts from resembling the stellar disk in the $3.6 \mu \mathrm{m}$ image to an ISM-like appearance at $8 \mu \mathrm{m}$. We assume conservatively that the $3.6 \mu \mathrm{m}$ emission is all due to stars and extrapolate this component to longer wavelengths using stellar population modeling from Starburst99 (Leitherer et al. 1999); the stellar spectral energy distribution (SED) longward of $2 \mu \mathrm{m}$ depends very little on star formation history or metallicity, as illustrated in Figure 2. The decomposition amounts to scaling the $3.6 \mu \mathrm{m}$ map by $0.596,0.399,0.232$, and 0.032 , respectively, for 4.5 , $5.8,8$, and $24 \mu \mathrm{m}$ and subtracting these from the observed maps pixel by pixel to yield what we refer to as the "dust maps" shown in Figure 1. Based on this decomposition, the $24 \mu \mathrm{m}$ map is probably $\geq 98 \%$ interstellar emission globally and $\geq 93 \%$ interstellar in the inner arcminute, where the stellar contribution is the greatest. The $8 \mu \mathrm{m}$ map is $\geq 81 \%$ interstellar emission.

This method potentially overestimates the dust component by underestimating the redder SED of very low mass stars. However the model reproduces adequately the $4.5 \mu \mathrm{m} / 3.6 \mu \mathrm{m}$ color of the nuclear stellar cluster, of the emission averaged in the inner arcminute (Fig. 2), and of the diffuse emission outside several disk $\mathrm{H}$ II regions.

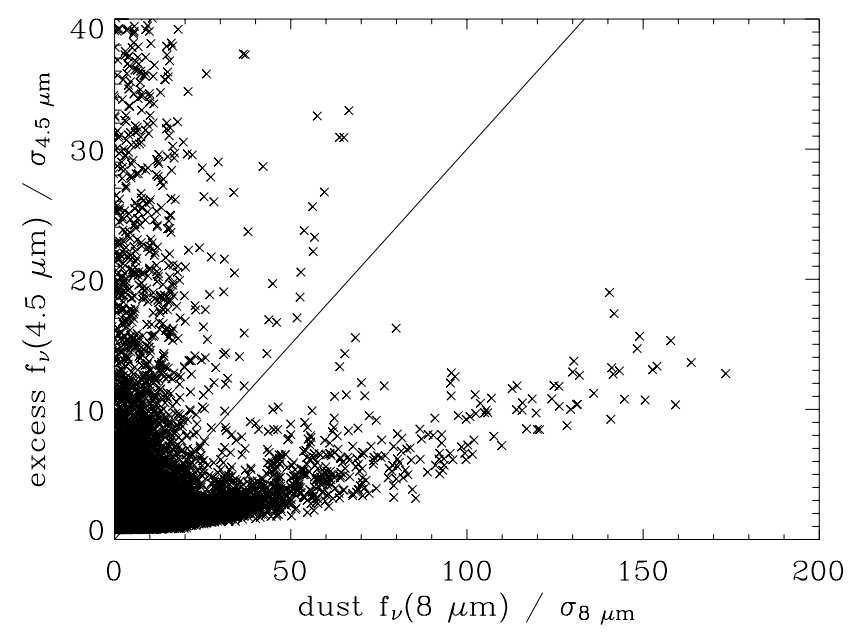

FIG. 3.- Scatter plot of excess $f_{\nu}(4.5 \mu \mathrm{m})$ vs. dust-only $f_{\nu}(8 \mu \mathrm{m})$, normalized by the noise in the original maps. Individual pixels are plotted, after convolution of all maps to a common angular resolution. The correlation branch was isolated by selecting pixels below the solid line (corresponding to Fig. $4 a$ ). The uncorrelated emission, extending to much higher brightnesses, was cut here for clarity. The scatter plot vs. $\mathrm{H} \alpha$, not included, shows a similar correlation branch, most pixels of which map into the $8 \mu \mathrm{m}$ correlation branch.

On the other hand, the method probably underestimates the dust component by assuming that the $3.6 \mu \mathrm{m}$ map contains only photospheric emission, ignoring evidence that the hot dust component stretches down to $\lambda<3 \mu \mathrm{m}$ (Bernard et al. 1994; Hunt et al. 2002; Lu et al. 2003). From the Infrared Space Observatory (ISO) data in Figure 6 of Lu et al. (2003), this underestimate may reach a factor of up to $2.6,10 \%-20 \%$, and $3 \%-4 \%$, respectively, at $4.5,5.8$, and $8 \mu \mathrm{m}$. In view of these opposing potential biases, our conservative approach should be reliable qualitatively for NGC 300, in spite of localized departures due to variations in stellar population colors or dust extinction.

\subsection{Interstellar Dust Emission}

In order to constrain the origin of the $4.5 \mu \mathrm{m}$ nonstellar emission, we identified pixels where the excess is above the $3 \sigma$ significance threshold in the $4.5 \mu \mathrm{m}$ "dust map" and examined their colors and locations. In scatter plots of these pixel values versus $\mathrm{H} \alpha$ or versus $8 \mu \mathrm{m}$ dust emission, a correlation branch is evident in both cases with a moderate slope in $f_{\nu}(4.5 \mu \mathrm{m}) / f_{\nu}(8 \mu \mathrm{m})$ and $f_{\nu}(4.5 \mu \mathrm{m}) / \mathrm{H} \alpha$ (Fig. 3), as well as a branch with a very steep, almost vertical slope. The pixels in the latter branch are strongly associated with the $3.6 \mu \mathrm{m}$ emission, including the brightest foreground star and a large number of point sources spread almost uniformly across the whole map. We use this excess map as a mask to efficiently remove foreground stars, with additional hand masking of residues around very bright stars.

On the other hand, the branches with moderate $4.5 \mu \mathrm{m} /$ $8 \mu \mathrm{m}$ and $4.5 \mu \mathrm{m} / \mathrm{H} \alpha$ ratios map into bright star formation regions, as might be expected (Fig. $4 a$ [Plate 2]). The global ratio $\nu f_{\nu}(4.5 \mu \mathrm{m}) / \nu f_{\nu}(8 \mu \mathrm{m})$ for the selected dust emission amounts to $\sim 4 \%$. However, when summing the pixels in starforming regions with large $4.5 \mu \mathrm{m}$ excess, dust alone accounts for $17 \%$ of the total $4.5 \mu \mathrm{m}$ emission, probably more if dust contribution to the $3.6 \mu \mathrm{m}$ emission were accounted for. Two uncertainties remain that will be addressed in future papers: Whether or not redder SEDs of young stars significantly affect the results in star-forming regions and whether the $4.5 \mu \mathrm{m}$ 
dust associates more closely with ionized regions or with photodissociation regions (PDRs).

The dust maps at 5.8 and $8 \mu \mathrm{m}$ should be dominated by the aromatic features in emission (AFEs) proposed by Puget \& Léger (1989) to explain IRAS data and well studied with ISO (Boulanger et al. 1998; Helou et al. 2000; Lu et al. 2003). The value and variability of the ratios between these bands are of considerable interest in constraining the physical state of the emitters (e.g., de Frees et al. 1993). Lu et al. (2003) found the AFE profiles among galaxies constant to within a measurement uncertainty of $15 \%-25 \%$ in the range $5-12 \mu \mathrm{m}$; the Spitzer images can extend this finding to much greater accuracy and could point to variations within disks on a variety of scales.

We find that $\nu f_{\nu}(5.8 \mu \mathrm{m}) / \nu f_{\nu}(8 \mu \mathrm{m})$ for dust emission alone is constant to within the uncertainties, with a value of $0.50 \pm$ $0.09(3 \sigma)$ over most of the area where surface brightness is above the $6 \sigma$ level in each of the 5.8 and $8 \mu \mathrm{m}$ dust maps.

\subsection{Comparison to $\mathrm{H} \alpha$}

Figure $4 c$ shows a comparison between $\mathrm{H} \alpha$ and $8 \mu \mathrm{m}$ maps at a resolution of $2(\mathrm{FWHM}) \sim 5^{\prime \prime} .15 \sim 50 \mathrm{pc}$. In the outer disk, several $\mathrm{H}$ II regions, as well as more complex structures, are visible in the $\mathrm{H} \alpha$ map, with the corresponding $8 \mu \mathrm{m}$ profile flatter and sometimes significantly offset from the $\mathrm{H} \alpha$ peak. In spite of the complex structure, Figures $4 c-4 d$ clearly show the $8 \mu \mathrm{m}$ emission highlighting the rims of $\mathrm{H}$ II regions. The ratio $8 \mu \mathrm{m} / \mathrm{H} \alpha$ goes up by a factor of $5-10$ from inside to just outside the $\mathrm{H}$ II region. This ratio may increase by another factor of 2-4 in diffuse regions, but this is uncertain because of limited sensitivity and inaccurate continuum subtraction in the $\mathrm{H} \alpha$ map. While this behavior is not a complete surprise, the Spitzer data are the first to show it so clearly in a nearby galaxy. Boulanger et al. (1990), Sellgren et al. (1990), Giard et al. (1994), and others have reported sharp boundaries to the AFEs, with peaks on molecular cloud surfaces outside ionized regions, and related this to a combination of UV excitation and photoprocessing of the AFE carriers. Helou et al. (2001) also speculated that AFEs arise primarily in PDRs based on physical arguments rather than direct observations. Inside the $\mathrm{H}$ II regions, the aromatics are depressed, probably because of destruction by the ionizing UV (Boulanger et al. 1990).

The sharp contrasts near $\mathrm{H}$ II regions between $8 \mu \mathrm{m}$ and $\mathrm{H} \alpha$ are only a small-scale property. Summing over scales larger than about $1 \mathrm{kpc}$, the ratio between the two tracers is constant to about $40 \%$. The global ratio of $8 \mu \mathrm{m}$ to unextincted $\mathrm{H} \alpha$ is roughly $30 \%$ smaller than the average measured in disks of spiral galaxies by Roussel et al. (2001a), which itself had a dispersion of $50 \%$ among disks. For this comparison, the total $\mathrm{H} \alpha+\left[\mathrm{N}_{\text {II }}\right]$ flux from Hoopes et al. (1996) was corrected for an extinction $A(\mathrm{H} \alpha)=0.37$ and an $\mathrm{H} \alpha /\left(\mathrm{H} \alpha+\left[\mathrm{N}_{\text {II }}\right]\right)$ ratio of 0.91 , averaged from values of individual $\mathrm{H}$ II regions in Webster \& Smith (1983). The $8 \mu \mathrm{m}$ IRAC flux was converted to $7 \mu \mathrm{m}$ flux in the ISO filter LW2, assuming the same spectral shape as in the disk of M83 (Roussel et al. 2001b), which was verified to reproduce accurately the $5.8 \mu \mathrm{m} / 8 \mu \mathrm{m}$ dust ratio of NGC 300.

The star formation rate in the disk of NGC 300 is $0.08-$ $0.11 M_{\odot} \mathrm{yr}^{-1}$ from the $8 \mu \mathrm{m}$ emission (as calibrated for solarmetallicity spiral disks by Roussel et al. 2001a) and $\sim 0.14 M_{\odot}$ $\mathrm{yr}^{-1}$ from $\mathrm{H} \alpha$ (as calibrated by Kennicutt 1998). The agreement points to $8 \mu \mathrm{m}$ under these conditions as a viable proxy for ionizing flux and therefore for the ongoing massive star formation rate. Since the association between $8 \mu \mathrm{m}$ and $\mathrm{H} \alpha$ is not at the atomic process level however, it is subject to geometric and other effects, and the use of $8 \mu \mathrm{m}$ as such a proxy is unlikely to be valid under all conditions.

Figures $4 e-4 f$ also show that the $24 \mu \mathrm{m}$ emission is more peaked than $8 \mu \mathrm{m}$ in star-forming regions. The spatial resolution is insufficient to distinguish whether PDRs or ionized regions are more closely associated with the $24 \mu \mathrm{m}$ peaks. This confirms earlier suggestions based on global SED analysis that $24 \mu \mathrm{m}$ traces heating by the youngest and most massive stars in a galaxy (Helou 2000).

\section{MID- TO FAR-INFRARED SED}

In order to study the SED at $\lambda \geq 8 \mu \mathrm{m}$, we identified 19 emission regions in the dust maps, most centered on a local peak (Fig. 5). After smoothing all maps to the resolution of $160 \mu \mathrm{m}$, we measured the emission of each region in a circular aperture of $2.5[\mathrm{FWHM}(160 \mu \mathrm{m})]=95^{\prime \prime} \sim 1 \mathrm{kpc}$ in diameter. The resulting photometry is plotted in Figure 5 as various color ratios versus $f_{\nu}(8 \mu \mathrm{m}) / f_{\nu}(24 \mu \mathrm{m})$ and compared to the ratios expected from pre-Spitzer model SEDs of galaxies (Dale \& Helou 2002). These are single-parameter models of dust emission integrated over whole galaxies and incorporate a power-law distribution of dust over heating intensities (Dale et al. 2001). The data and model agree in the trends, although there are systematic offsets in the values. These offsets are most simply understood as a systematic discrepancy of $\sim 30 \%$ at $160 \mu \mathrm{m}$ and $\sim 15 \%$ at $70 \mu \mathrm{m}$ between model and measurement, assuming reliable photometry at 8 and $24 \mu \mathrm{m}$. In view of the simplicity of the models, photometric uncertainties (especially nonlinearity effects), and source extent, the agreement between model and data is quite satisfactory and suggests that beyond a certain scale portions of galaxies may approach full galaxies in their behavior.

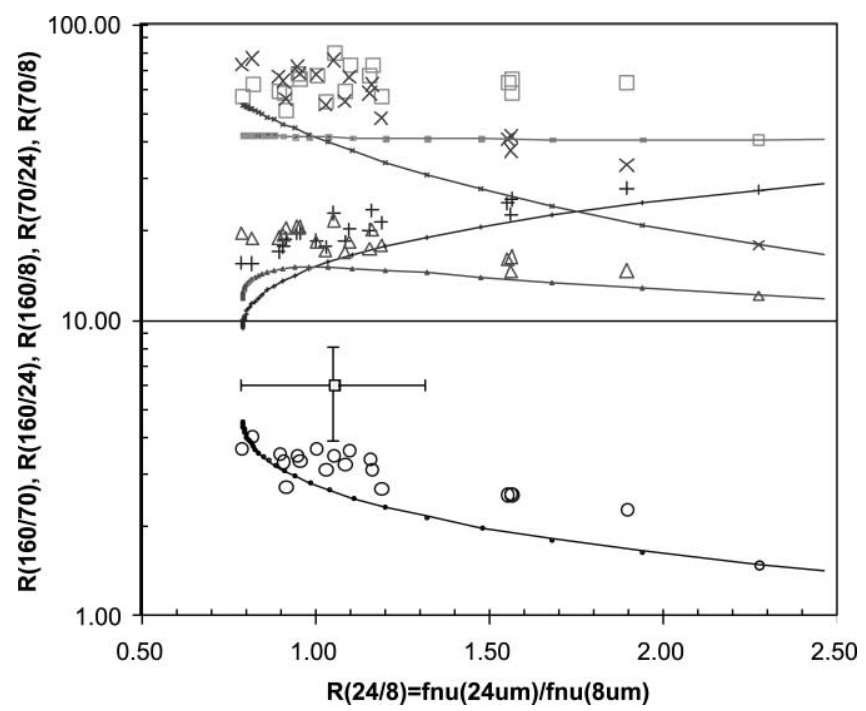

FIG. 5.-Color-color plot for all Spitzer bands at $\lambda \geq 8 \mu \mathrm{m}$, for the regions measured with an aperture corresponding to $\sim 1 \mathrm{kpc}$ in the plane of NGC 300, with the Dale \& Helou (2002) model predictions superposed as curves. Each curve has one symbol at $x=2.28$ to indicate the corresponding color ratio. We corrected the $8 \mu \mathrm{m}$ fluxes by a factor of $(0.69)^{1 / 2}$ since the aperture is intermediate in size between the nominal point-source aperture and infinitely extended scales. The one point with error bars illustrates the uncertainties of $25 \%$ on the $x$-axis and $35 \%$ on the $y$-axis. Squares are for $160 \mu \mathrm{m} / 8 \mu \mathrm{m}$, crosses are for $160 \mu \mathrm{m} / 24 \mu \mathrm{m}$, plus signs are for $70 \mu \mathrm{m} / 8 \mu \mathrm{m}$, triangles are for $70 \mu \mathrm{m} / 24 \mu \mathrm{m}$, and circles are for $160 \mu \mathrm{m} / 70 \mu \mathrm{m}$. The systematic offset at the longer wavelength bands is discussed in the text. [See the electronic edition of the Journal for a color version of this figure.] 
The $160 \mu \mathrm{m}$, and to a lesser extent the $70 \mu \mathrm{m}$, maps display a strikingly large halo that echoes several features of the $\mathrm{H} \mathrm{I}$ envelope. It is unclear at this point whether this reflects unusually cold material in the outer disk or whether the larger $160 \mu \mathrm{m}$ beam is simply more sensitive to low surface brightness features.

\section{SUMMARY}

The Spitzer Space Telescope data allow us more clearly than ever to dissect the interstellar dust emission components in NGC 300 and to relate them to star formation. The $8 \mu \mathrm{m}$ AFE highlights the rims of the $\mathrm{H}$ II regions defined by $\mathrm{H} \alpha$, confirming that it is more closely associated with PDRs than with ionized regions. When averaging over regions larger than $\sim 1 \mathrm{kpc}$, the ratio of $\mathrm{H} \alpha$ to AFE emission in NGC 300 is consistent with the disk value observed in other galaxies. This confirms AFE as a convenient massive star formation rate estimator in disks of galaxies. The mid-infrared spectral signature of the AFE is invariant as measured by the ratio $f_{\nu}(5.8 \mu \mathrm{m}) / f_{\nu}(8 \mu \mathrm{m})$, which is essentially constant at $\sim 50 \mathrm{pc}$ resolution at the $\sim 20 \%(3 \sigma)$ measurement accuracy over most of the disk of NGC 300 . The $24 \mu \mathrm{m}$ emission is more strongly peaked than $8 \mu \mathrm{m}$ in star-forming regions. Interstellar dust emission at $4.5 \mu \mathrm{m}$ is confirmed at about the expected amplitude; for the first time, its spatial distribution associates it clearly with star-forming regions. The data cannot distinguish between PDRs and $\mathrm{H}$ II regions as the primary origin for either 4.5 or $24 \mu \mathrm{m}$ dust emission. The SED models of Dale \& Helou (2002) apply to approximately kilparsec-sized portions in the disk to within the current photometry and calibration uncertainties of $20 \%-30 \%$.

The Spitzer Space Telescope is operated by the Jet Propulsion Laboratory, California Institute of Technology, under contract with the National Aeronautics and Space Administration. This research has made use of the NASA/IPAC Extragalactic Database, which is operated by JPL/Caltech, under contract with NASA.
Bernard, J. P., Boulanger, F., Désert, F. X., Giard, M., Helou, G., \& Puget, J. L. 1994, A\&A, 291, L5

Boulanger, F., Boissel, P., Cesarsky, D., \& Ryter, C. 1998, A\&A, 339, 194

Boulanger, F., Falgarone, E., Puget. J.-L., \& Helou, G. 1990, ApJ, 364, 136

Butler, D. J., Martinez-Delgado, D., \& Brandner, W. 2004, AJ, 127, 1472

Dale, D. A., \& Helou, G. 2002, ApJ, 576, 159

Dale, D. A., Helou, G., Contursi, A., Silbermann, N., \& Kolhatkar, S. 2001, ApJ, 549, 215

de Frees, D. J., Miller, M. D., Talbi, D., Pauzat, F., \& Ellinger, Y. 1993, ApJ, 408, 530

Deharveng, L., Caplan, J., Lequeux, J., Azzopardi, M., Breysacher, J., Tarenghi, M., \& Westerlund, B. 1988, A\&AS, 73, 407

Engelbracht, C. W., et al. 2004, ApJS, 154, 248

Fazio, G., et al. 2004, ApJS, 154, 10

Freedman, W. L., Madore, B. F., Hawley, S. L., Horowitz, I. K., Mould, J., Navarrete, M., \& Sallmen, S. 1992, ApJ, 396, 80

Giard, M., Bernard, J. P., Lacombe, F., Normand, P., \& Rouan, D. 1994, A\&A, 291, 239

Gordon, K. D., et al. 2004, PASP, submitted

Helou, G. 2000, in Infrared Astronomy: Today and Tomorrow, ed. F. Casoli, J. Lequeux, \& F. David (Springer: Berlin), 337

\section{EFERENCES}

Helou, G., Lu, N. Y., Werner, M. W., Malhotra, S., \& Silbermann, N. A. 2000, ApJ, 532, L21

Helou, G., Malhotra, S., Hollenbach, D. J., Dale, D. A., \& Contursi, A. 2001, ApJ, 548, L73

Hoopes, C. G., Walterbos, R. A., \& Greenawalt, B. E. 1996, AJ, 112, 1429

Hunt, L. K., Gio vanandi, C., \& Helou, G. 2002, A\&A, 394, 873

Kennicutt, R. C. 1998, ARA\&A, 36, 189

Larsen, S. S., \& Richtler, T. 1999, A\&A, 345, 59

Leitherer, C., et al. 1999, ApJS, 123, 3

Lu, N., et al. 2003, ApJ, 588, 199

Pannuti, T. G., Duric, N., Lacey, C. K., Goss, W. M., Hoopes, C. G., Walterbos, R. A. M., \& Magnor, M. A. 2000, ApJ, 544, 780

Puche, D., Carignan, C., \& Bosma, A. 1990, AJ, 100, 1468

Puget, J. L., \& Léger, A. 1989, ARA\&A, 27, 161

Rieke, G. H., et al. 2004, ApJS, 154, 25

Roussel, H., Sauvage, M., Vigroux, L., \& Bosma, A. 2001a, A\&A, 372, 427

Roussel, H., et al. 2001b, A\&A, 369, 473

Sellgren, K., Tokunaga, A. T., \& Nakada, Y. 1990, ApJ, 349, 120

Thuan, T. X., \& Sauvage, M. 1992, A\&AS, 92, 749

Webster, B. L., \& Smith, M. G. 1983, MNRAS, 204, 743

Werner, M. W., et al. 2004, ApJS, 154, 1 


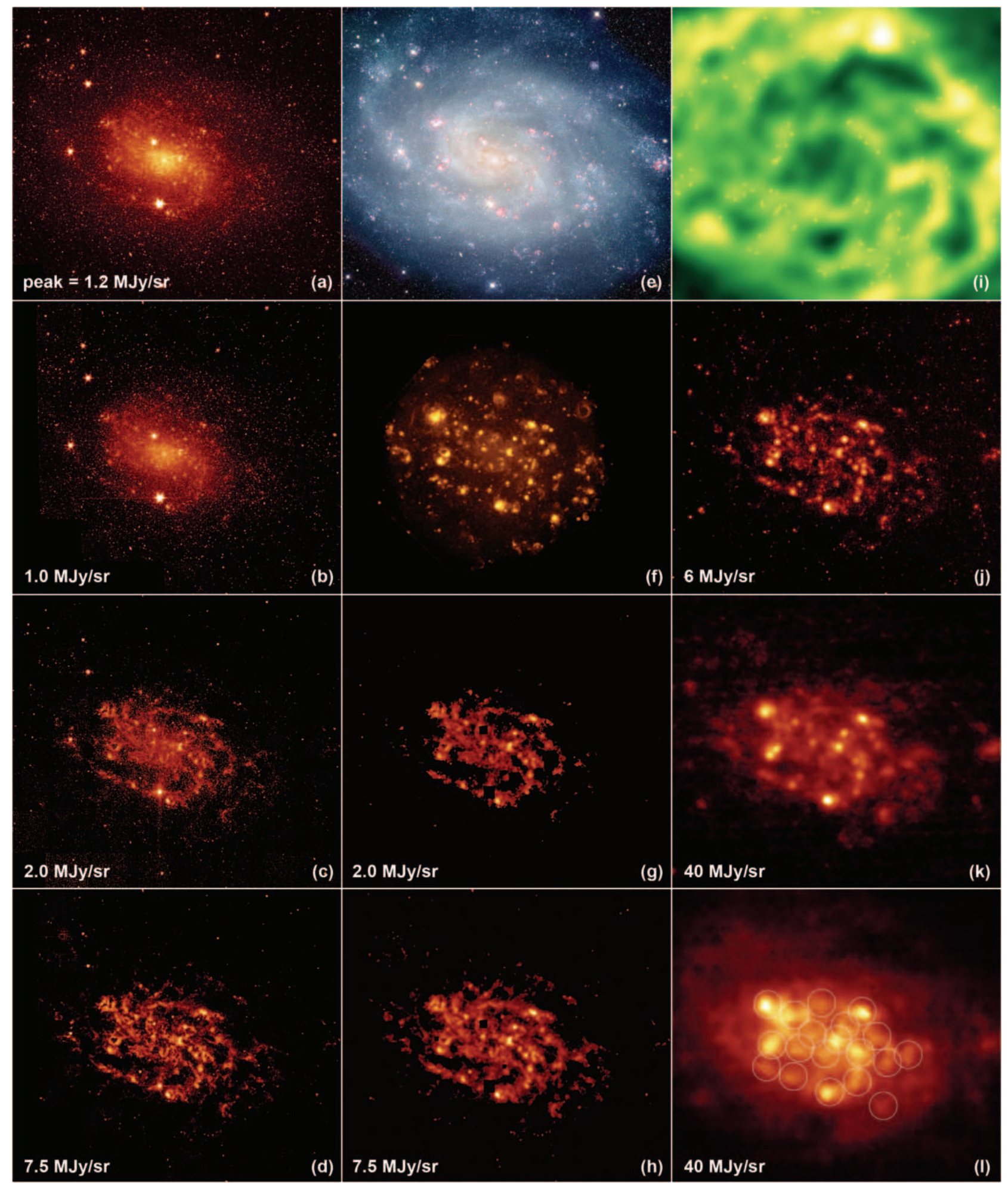

FIG. 1.-Images of NGC 300 obtained with Spitzer and other sources. The leftmost column shows observed maps at (a) $3.6 \mu \mathrm{m},(b) 4.5 \mu \mathrm{m},(c) 5.8 \mu \mathrm{m}$, and $(d)$ $8 \mu \mathrm{m}$. The center column shows (e) a $B V R \mathrm{H} \alpha$ composite image (credit MPG/ESO) in which $B$ is coded in blue, $V$ in green, and $R$ and $\mathrm{H} \alpha$ in red; $(f)$ the $\mathrm{H} \alpha$ map described in $\S 2 ;(g)$ a dust map at $5.8 \mu \mathrm{m}$; and $(h)$ a dust map at $8 \mu \mathrm{m}$. The rightmost column shows $(i)$ the H I map from Puche et al. (1990) and observed maps at $(j)$ $24 \mu \mathrm{m},(k) 70 \mu \mathrm{m}$, and $(l) 160 \mu \mathrm{m}$, with photometric apertures used in Fig. 5 superposed. Each frame contains the peak surface brightness in the backgroundsubtracted image; all images are in logarithmic scale, except the $160 \mu \mathrm{m}$ image, which is linear. North is about $40^{\circ}$ clockwise from vertical up, and each frame is approximately $20^{\prime}$ on a side. 

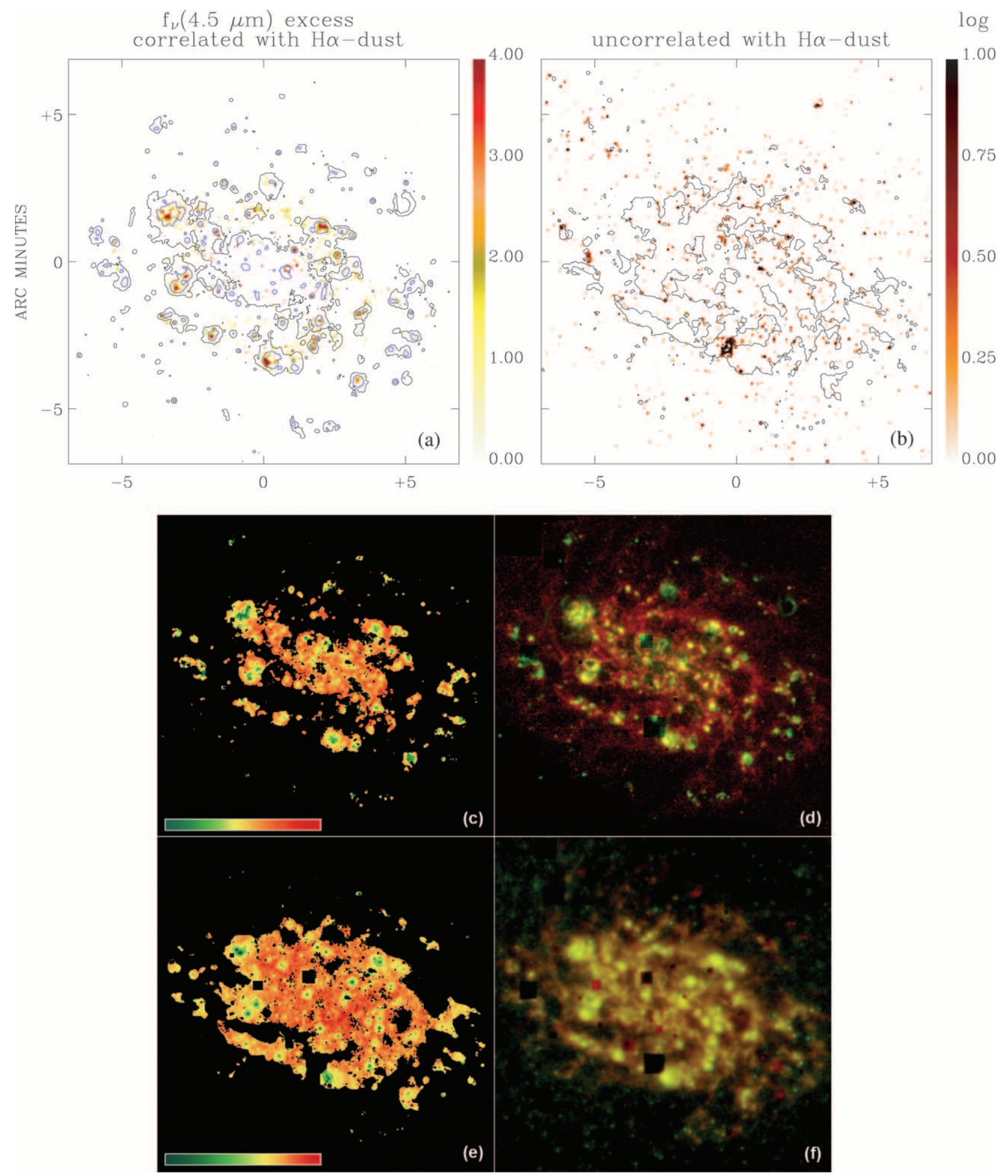

FIG. 4.-Top: Distribution of excess $4.5 \mu \mathrm{m}$ emission, $f_{\nu}^{e}(4.5 \mu \mathrm{m})$, in units of the noise in the initial $4.5 \mu \mathrm{m}$ map, $\sigma_{4.5}$. $(a)$ Part of $f_{\nu}^{e}(4.5 \mu \mathrm{m})$ that is correlated with $8 \mu \mathrm{m}$ and $\mathrm{H} \alpha$ [950 pixels with $\left.3 \sigma_{4.5}<f_{\nu}^{e}(4.5 \mu \mathrm{m})<19 \sigma_{4.5}\right]$; $(b)$ part of $f_{\nu}^{e}(4.5 \mu \mathrm{m})$ that is not correlated with $8 \mu \mathrm{m}$ nor with $\mathrm{H} \alpha$, in logarithmic scale [4583 pixels with $3 \sigma_{4.5} f_{\nu}^{e}(4.5 \mu \mathrm{m})<8150 \sigma_{4.5}$ ]. The contours are $(a) \mathrm{H} \alpha$ isophotes and $(b)$ the $4 \sigma$ isophote of the $8 \mu \mathrm{m}$ dust map. Bottom: Comparison of H $\alpha$ and dust emission: (c) $\log \left[f_{\nu}(8 \mu \mathrm{m}) / \mathrm{H} \alpha\right](95 \%$ of pixels span 1.2 dex), showing low ratios inside prominent $\mathrm{H}$ II regions; $(d) 8 \mu \mathrm{m}($ red $)$ and $\mathrm{H} \alpha$ (green) composite image; $(e) \log \left[f_{\nu}(8 \mu \mathrm{m}) / f_{\nu}(24 \mu \mathrm{m})\right](95 \%$ of pixels span $0.56 \mathrm{dex}$, showing again the distinctly low color ratios of H II regions; $(f) 8 \mu \mathrm{m}(r e d)$ and $24 \mu \mathrm{m}(g r e e n)$ composite image. 